\title{
Application of a new High-Pressure Freezing Apparatus
}

\author{
H. Krisp, ${ }^{*}$ G. vonWichert, ${ }^{* *}$ T. Seufferlein, ${ }^{* *}$ and P. Walther* \\ * Central Electron Microscopy Laboratory, University of Ulm, 89069 Ulm, Germany \\ ** Department of Internal Medicine I, University of Ulm, 89071 Ulm, Germany
}

High pressure freezing allows for cryo-fixation of biological samples from a defined physiological state, up to $200 \mu \mathrm{m}$ thick (depending on the water content of the sample) without formation of structure destroying ice crystals. Whereas this $200 \mu \mathrm{m}$ may represent a basic physical limitation of the technique, improvements are requested regarding faster and more careful sampling before freezing and freezing at a defined physiological state of the sample. In addition, the geometry of sample holders should allow for a versatile follow-up preparation, such as freeze substitution and immunolabelling as well as freeze-fracturing and cryo-sectioning for TEM and cryo-SEM [1].

We tried to achieve these goals by using the newly designed high-pressure freezer Compact HPF-01 (Wohlwend GmbH, Switzerland, distributed by TECHNOTRADE International, Inc).

The features of the new Compact HPF-01 are:

-Different sample holders, including the standard aluminum platelets that are used for small tissue pieces and for thin cellulose capillary tubes [2] for freeze substitution and immunolabelling, copper sandwich platelets (similar to those used for propane jet freezing) for freeze-fracturing and freeze-etching [1], and small aluminum tubes for native cryo sectioning.

-Optional freezing without ethanol, due to the very fast pressure build-up. (Ethanol is usually used to synchronize pressure build-up and cooling; ethanol residues on the sample holders can, however, cause problems during freeze fracturing).

-The size of the machine is reduced to $800 \times 700 \times 1400 \mathrm{~mm}$. This significantly reduces weight and liquid nitrogen consumption without affecting the cooling rate.

For this study we prepared pancreatic cancer cells in culture, grown as a monolayer on special 3 mm sapphire discs [3] (distributed by M. Wohlwend GmbH, Switzerland) that fit into the $3 \mathrm{~mm}$ aluminum platelets used for high-pressure freezing. After high-pressure freezing, samples were freeze-substituted in acetone containing $5 \%$ of water to increase membrane visibility [4]. The sample in Figs. 1 and 2 was substituted with osmium and embedded in Epon; the sample in Figs. 3 and 4 was substituted without osmium and embedded in LR Gold. The combination of fluorescence light microscopy and electron microscopy on the very same section allows for a very accurate localization of the GFP signal. It remains to be shown, whether this method is also suitable for systems with lower GFP expression.

[1] P. Walther, J. Microsc. 212, (2003) p. 34-43.

[2] H. Hohenberg et al., J. Microsc. 175, (1994) p. 34-43

[3] M. Müller, ETH Zürich, personal communication

[4] P. Walther and A. Ziegler, J Microsc 208 (2002) 3-10 

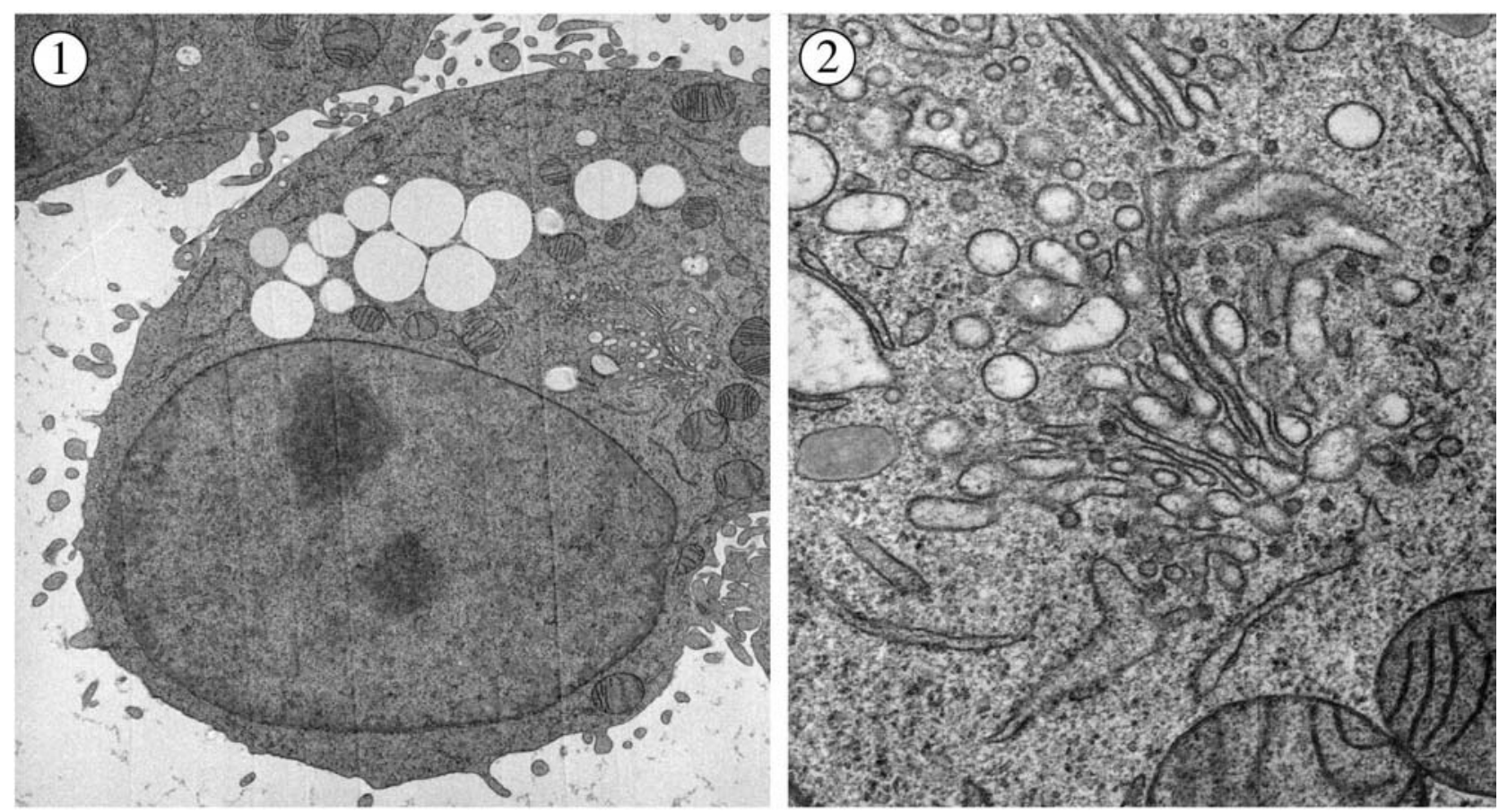

(3)

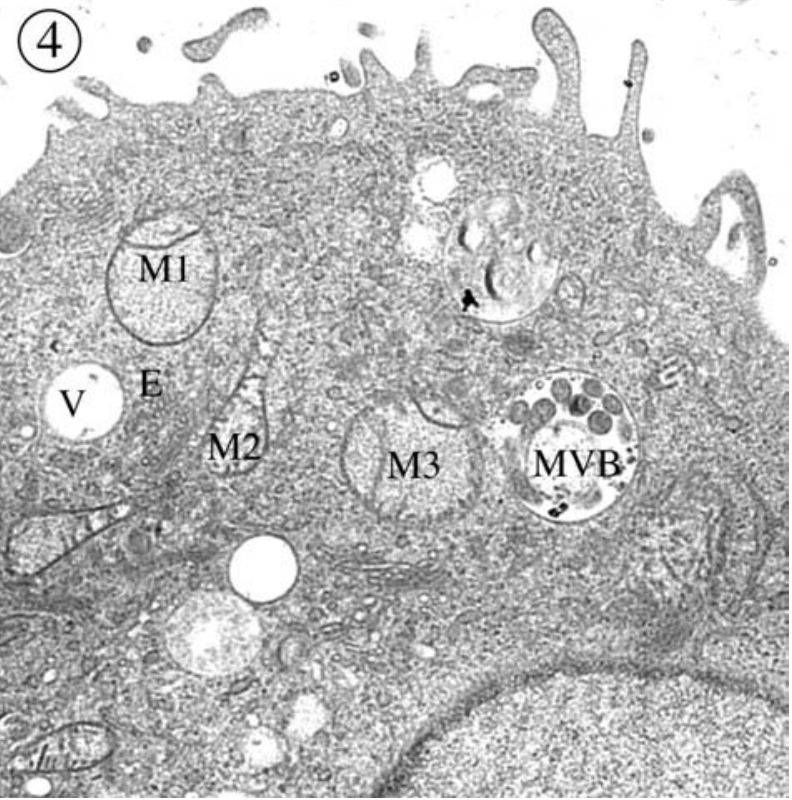

FIG 1 shows a high pressure frozen AR42J cell (exocrine rat pancreatic tumor cell) FIG 2 is a higher magnification of the Golgi apparatus. Note the complete absence of ice crystal segregation patterns, indicating good freezing. Figs. 3 and 4. The same area of the same section of a transfected BON cell, with GFP as control vector for PKD 2 expression. The EM grid was first analyzed in the fluorescent light microscope (Fig. 3) to show the GFP distribution, which is still well visible after high-pressure freezing and freeze substitution, and then in the TEM to visualize the ultrastructure. As expected, the GFP signal is located in the cytoplasm, but not in mitochondria (M) or vesicles (V) or multivesicular bodies (MVB). 